\title{
Transcriptome profiling of pluripotent pig embryonic stem cells originating from uni- and biparental embryos
}

\author{
Kwang-Hwan Choi ${ }^{1 \dagger}$, Dong-Kyung Lee ${ }^{1 \dagger}$, Jong-Nam Oh${ }^{1}$, Seung-Hun Kim ${ }^{1}$ Mingyun Lee ${ }^{1}$, Sung Woo Kim² \\ and Chang-Kyu Lee ${ }^{1,3^{*}}$
}

\begin{abstract}
Objectives: Pig pluripotent stem cells have tremendous potential because the pig is a valuable animal as both an agricultural resource and as a preclinical model of human therapy. To date, a lack of understanding of pig pluripotency has inhibited the derivation of embryonic stem cells (ESCs) and transgene-free induced pluripotent stem cells. Therefore, there has been no accessible or reliable transcriptome data for researching the genuine pig pluripotency network. Our previous study isolated authentic pig ESCs, which had teratoma-forming and direct differentiation ability, that were derived by activating the FGF2, ACTIVIN A, and WNT pathways. Here, we aimed to provide detailed information on transcriptome data of the newly derived pig ESCs and perform a comparative analysis with pig preimplantation embryo transcriptomes in a public database.
\end{abstract}

Data description: The transcriptome data of ESCs derived from in vitro fertilized and parthenogenetic embryos were generated by HiSeq 2500. Then, differentially expressed genes (DEGs) from each sample were compared with fibroblasts, and gene expression profiling was carried out for comparative analysis. Our data, as the first transcriptome dataset for genuine pig pluripotent cells, could be a general reference for explaining the molecular mechanism of species-specific pluripotency and improving understanding of the embryo development of domestic animals.

Keywords: Pig embryonic stem cells, RNA-seq, Transcriptome, In vitro fertilized embryo, Parthenogenetic embryo, Preimplantation embryo

\section{Objective}

The pig is a valuable animal as a preclinical model of human therapy as well as an agricultural resource. Recent studies have found that pig germ cell development more closely resembles the molecular features of human germ cell specification than that of the mouse [1] and that the pig is an ideal candidate for interspecies-chimera formation with human pluripotent stem cells (PSCs) to provide

\footnotetext{
*Correspondence: leeck@snu.ac.kr

${ }^{\dagger}$ Kwang-Hwan Choi and Dong-Kyung Lee Co-first authors

${ }^{1}$ Department of Agricultural Biotechnology, Animal Biotechnology Major, and Research Institute of Agriculture and Life Science, Seoul National University, Seoul 08826, South Korea

Full list of author information is available at the end of the article
}

a source for xenotransplantation of organs [2]. Accordingly, research into pig PSCs such as embryonic stem cells (ESCs) and induced pluripotent stem cells (iPSCs) is required for understanding their comparative developmental biology and medical/agricultural applications. However, to date, no stable and well-characterized pig PSCs have been derived due to a lack of understanding of pig pluripotency networks and suitable culture conditions $[3,4]$. Instead, ES-like cells, having no in vivo differentiation ability, and transgene-dependent partially reprogrammed iPSCs have been derived and researched over the last few decades [5]. Recently, through systemic screening of key pathways for pig pluripotency circuitry, two independent research groups, including our group, 
reported two different types of genuine pig PSCs derived from embryos that have in vivo and in vitro differentiation potentials $[6,7]$.

Transcriptome data of PSCs derived from embryos and somatic cells are considered to be valuable tools for the investigation of pluripotency. Transcriptome analysis of pluripotent cells provides a comprehensive biological insight into developmental cues that we couldn't acquire before through traditional gene analysis techniques. Pluripotent cells originate during the preimplantation period of embryo development and the reprogramming process of somatic cells [8]. Accordingly, this study aimed to offer comprehensive information and improve the reusability of an RNA-sequencing (seq) data set of pig ESCs derived from in vitro-fertilized and parthenogenetic embryos.

\section{Data description}

\section{Cell preparation and RNA extraction}

Pig ESCs were cultured with feeder cells made of mitotically inactivated mouse embryonic fibroblasts to maintain their pluripotency in vitro. Therefore, the feeder cells needed to be removed from the ESC samples to obtain a highly reliable RNA-seq data from a homogenous population of the undifferentiated pig ESCs. To exclude these contaminating cells, the pig ESCs were sorted by a MACS system using an antibody against SSEA1, which is highly expressed on the surface of undifferentiated pig ESCs [6]. The RNA integrity number (RIN) and 28S/18S
rRNA ratio were determined by the Agilent 2100 BioAnalyzer to estimate the integrity and degradation level of the extracted RNA samples. The RIN values and 28S/18S rRNA ratio of the samples used in this study were greater than 9.2 and 1.9, respectively, which indicated that the samples for RNA-seq have high integrity and a low degradation level (Table S1, Table 1; All of the data files described in this data note is summarized in Table 1).

\section{Quality control assessment}

As evaluated by the Agilent 2100 BioAnalyzer, all of the libraries used in this study have a uniform and constant size of approximately $380 \mathrm{bp}$ and passed quality control to be used for sequencing. Then, base call quality scores were calculated by the FastQC program to assess the quality of the raw sequencing data of the cDNA libraries (Dataset 1). Low-quality reads were filtered according to the criteria as described in the Methodology (Supplementary file 1). After filtration of low-quality reads, as shown in the box plot distributions of Figure S1, the quality scores across all bases were in the high confidence range. The filtered sequence reads and previously reported data for pig preimplantation embryos [9] were aligned to the pig reference genome (Sscrofa10.2) with 80.5-92.2\% mapping rates (Table S2). After the mapping, gene expression levels were calculated by the HTSeq-count program (Fig. S2a, Dataset 2). These data were visualized by the R program as an MDS plot, which

Table 1 Overview of data files/data sets

\begin{tabular}{|c|c|c|c|}
\hline Label & Name of data file/data set & File types (file extension) & $\begin{array}{l}\text { Data repository and identifier (DOI } \\
\text { or Accession Number) }\end{array}$ \\
\hline Supplementary file 1 & Methodology description & Document file (.pdf) & $\begin{array}{l}\text { Figshare (https://doi.org/10.6084/m9.figsh } \\
\text { are.10298324.v2) }\end{array}$ \\
\hline Dataset 1 & $\begin{array}{l}\text { Raw FASTQ files for the RNA-seq. of pig embry- } \\
\text { onic stem cells }\end{array}$ & Fastq files (.fastq) & NCBI Gene Expression Omnibus (GSE120031) \\
\hline Dataset 2 & $\begin{array}{l}\text { List of normalized gene expression level (FPKM; } \\
\text { ESCs and Embryos) }\end{array}$ & Spreadsheet (.x|sx) & $\begin{array}{l}\text { Figshare (https://doi.org/10.6084/m9.figsh } \\
\text { are.10298324.v2) }\end{array}$ \\
\hline Dataset 3 & $\begin{array}{l}\text { Lists of differentially expressed genes (FC; ESCs } \\
\text { and Embryos) }\end{array}$ & Spreadsheet (.xIsx) & $\begin{array}{l}\text { Figshare (https://doi.org/10.6084/m9.figsh } \\
\text { are.10298324.v2) }\end{array}$ \\
\hline Table S1 & Summary of cell samples for RNA-seq. & Spreadsheet (.xIsx) & $\begin{array}{l}\text { Figshare (https://doi.org/10.6084/m9.figsh } \\
\text { are.10298324.v2) }\end{array}$ \\
\hline Table S2 & Summary of alignment statistics for RNA-seq. & Spreadsheet (.xIsx) & $\begin{array}{l}\text { Figshare (https://doi.org/10.6084/m9.figsh } \\
\text { are.10298324.v2) }\end{array}$ \\
\hline Table S3 & $\begin{array}{l}\text { Number of differentially expressed genes (DEGs) } \\
\text { compared with fibroblasts }\end{array}$ & Spreadsheet (.xIsx) & $\begin{array}{l}\text { Figshare (https://doi.org/10.6084/m9.figsh } \\
\text { are.10298324.v2) }\end{array}$ \\
\hline Figure S1 & Quality assessment of raw FASTQ files & Document file (.pdf) & $\begin{array}{l}\text { Figshare (https://doi.org/10.6084/m9.figsh } \\
\text { are.10298324.v2) }\end{array}$ \\
\hline Figure S2 & $\begin{array}{l}\text { Workflow of the transcriptome analysis and the } \\
\text { results of comparative analysis }\end{array}$ & Document file (.pdf) & $\begin{array}{l}\text { Figshare (https://doi.org/10.6084/m9.figsh } \\
\text { are.10298324.v2) }\end{array}$ \\
\hline Figure S3 & $\begin{array}{l}\text { Gene expression profiling in pig ESC lines and } \\
\text { preimplantation embryos }\end{array}$ & Document file (.pdf) & $\begin{array}{l}\text { Figshare ( https://doi.org/10.6084/m9.figsh } \\
\text { are.10298324.v2) }\end{array}$ \\
\hline
\end{tabular}


demonstrated four clusters including fibroblasts, ESCs, epiblasts and inner cell mass (ICM) (Fig. S2b).

\section{Comparative analysis of gene expression}

The R package TTC was used for differentially expressed genes (DEGs) analysis (Dataset 3). DEGs of embryos and ESCs compared with fibroblasts were measured based on cutoffs of fold change $>2.5$ and a $p$ value $<0.05$ (Table S3 and Fig. S2c). Compared with fibroblasts, 2189, 2450 and 2327 genes were upregulated in PG-ES-3, PG-ES-7 and IVF-ES-11, respectively, and 1887 genes were commonly expressed in the three different ESC lines (Fig. S2d). Moreover, upregulated DEGs in the embryo samples were compared with genes commonly upregulated in ESCs. As a result, 200 genes were commonly expressed in all samples, and 936, 542, 269 and 283 genes were uniquely expressed in ESCs, day 7-8 ICM, day 10-11 epiblasts and day 12-13 epiblasts, respectively (Fig. S2e). Finally, expression of genes related to pluripotency, cellular signaling and lipid metabolism were analyzed (Fig. S3). Pig ESCs had more similarity with epiblasts than the ICM, indicating similar patterns observed in a previous study [6].

\section{Limitations}

The sample size of the experimental groups was not sufficient for reliable comparative analysis. Gender and sequencing platforms were not matched between ESCs and embryo samples.

\section{Abbreviations \\ PSCs: Pluripotent stem cells; ESCs: Embryonic stem cells; iPSCs: Induced pluri- potent stem cells; ICM: Inner cell mass; DEGs: Differentially expressed genes; RIN: RNA integrity number; RNA-seq: RNA-sequencing.}

\section{Acknowledgements}

Not applicable.

\section{Authors' contributions}

KHC and DKL performed, analyzed and interpreted all cell experiments. JNO, SHK and ML performed in vitro production of embryos. KHC, DKL and SWK performed, analyzed and interpreted the RNA-sequencing analysis. KHC, DKL and CKL conceived all of the experiments and wrote the manuscript, with contributions from all other authors. All authors read and approved the final manuscript.

\section{Funding}

This work was supported by the BK21 Plus Program and the National Research Foundation of Korea (NRF) Grant funded by the Korea government (NRF2019R1 C1C1004514), and partially supported by Grants from the Agenda Program of Rural Development Administration, Republic of Korea (No. PJ01362402). Funding is used to cover laboratory expenses, sample preparation, and sequencing. These funding agencies have no role in research design, data collection, analysis of results, or manuscript writing.

\section{Availability of data and materials}

Raw FASTQ files for the RNA-seq were deposited in the GEO database under accession number GSE1 20031 (https://identifiers.org/geo:GSE120031) [10]. The methodology description and processed data (dataset 1, dataset 2, Tables S1-S3, and Figures S1-S3) are available in Figshare (https://doi.org/10.6084/m9.figsh are.10298324.v2) [11].

\section{Ethics approval and consent to participate}

The care and experimental use of pigs and mice were approved by the Institutional Animal Care and Use Committee (IACUC) at Seoul National University (Approval nos.: SNU-160120-9-1, SNU-161114-3-1, and SNU-170223-2-1).

\section{Consent for publication}

Not applicable.

\section{Competing interests}

The authors declare that they have no competing interests.

\section{Author details}

${ }^{1}$ Department of Agricultural Biotechnology, Animal Biotechnology Major, and Research Institute of Agriculture and Life Science, Seoul National University, Seoul 08826, South Korea. ${ }^{2}$ Animal Genetic Resources Research Center, National Institute of Animal Science, RDA, Namwon, Jeollabuk-do 55717, South Korea. ${ }^{3}$ Institute of Green Bio Science and Technology, Seoul National University, Pyeong Chang, Kangwon-do 25354, South Korea.

Received: 15 November 2019 Accepted: 27 February 2020

Published online: 03 August 2020

\section{References}

1. Kobayashi T, Zhang H, Tang WWC, Irie N, Withey S, Klisch D, Sybirna A, Dietmann S, Contreras DA, Webb R, et al. Principles of early human development and germ cell program from conserved model systems. Nature. 2017;546(7658):416-20.

2. Wu J, Platero-Luengo A, Sakurai M, Sugawara A, Gil MA, Yamauchi T, Suzuki K, Bogliotti YS, Cuello C, Morales Valencia M, et al. Interspecies chimerism with mammalian pluripotent stem cells. Cell. 2017;168(3):473-486 e415.

3. Yuan Y. Capturing bovine pluripotency. Proc Natl Acad Sci USA. 2018;115(9):1962-3.

4. Choi KH, Lee CK. Pig pluripotent stem cells as a candidate for biomedical application. J Anim Reprod Biotechnol. 2019;34(3):139-47.

5. Choi KH, Park JK, Son D, Hwang JY, Lee DK, Ka H, Park J, Lee CK. Reactivation of endogenous genes and epigenetic remodeling are barriers for generating transgene-free induced pluripotent stem cells in pig. PLOS ONE. 2016;11(6):e0158046.

6. Choi KH, Lee DK, Kim SW, Woo SH, Kim DY, Lee CK. Chemically defined media can maintain pig pluripotency network in vitro. Stem Cell Rep. 2019. https://doi.org/10.1016/j.stemcr.2019.05.028.

7. Gao X, Nowak-Imialek M, Chen X, Chen D, Herrmann D, Ruan D, Chen ACH, Eckersley-Maslin MA, Ahmad S, Lee YL, et al. Establishment of porcine and human expanded potential stem cells. Nat Cell Biol. 2019;21 (6):687-99.

8. Plath K, Lowry WE. Progress in understanding reprogramming to the induced pluripotent state. Nat Rev Genet. 2011;12(4):253-65.

9. Secher JO, Ceylan A, Mazzoni G, Mashayekhi K, Li T, Muenthaisong S, Nielsen $T T, L i D, L i S$, Petkov S, et al. Systematic in vitro and in vivo characterization of leukemia-inhibiting factor- and fibroblast growth factor-derived porcine induced pluripotent stem cells. Mol Reprod Dev. 2017;84(3):229-45. https:// doi.org/10.1002/mrd.22771.

10. Choi KH, Lee DK, Kim SW, Lee CK. Derivation of authentic porcine embryonic stem cells using defined culture conditions. Gene Expression Omnibus. 2019. https://identifiers.org/geo:GSE120031. Accessed 08 Jul 2019.

11. Choi KH, Lee DK, Oh JN, Kim SH, Lee M, Kim SW, et al. Supplementary files for transcriptome profiling of pluripotent pig embryonic stem cells originating from uni- and biparental embryos. figshare. 2019. https://doi. org/10.6084/m9.figshare.10298324.v2.

\section{Publisher's Note}

Springer Nature remains neutral with regard to jurisdictional claims in published maps and institutional affiliations. 